\title{
Effects of heavy quarks on the deconfinement phase transition
}

Pok Man Lo*ab

${ }^{a}$ GSI, Planckstr. 1, D-64291 Darmstadt, Germany

${ }^{b}$ Institute of Theoretical Physics, University of Wroclaw, PL-50204 Wroctaw, Poland

E-mail: pmlo@gsi.de

The influence of heavy quarks on color deconfinement is examined within an effective model of gluons interacting with dynamical quarks. As the quark mass decreases, the strength of the explicit Z(3) symmetry breaking grows, and the first-order phase transition in the pure $\mathrm{SU}(3)$ gauge theory ends in a critical end point. The nature of this critical end point is explored by studying the fluctuations of the Polyakov loop, quantified by the corresponding susceptibilities. We argue that the ratio of Polyakov loop susceptibilities characterizes the onset of deconfinement in QCD.

9th International Workshop on Critical Point and Onset of Deconfinement

17-21 November, 2014

ZiF (Center of Interdisciplinary Research), University of Bielefeld, Germany

${ }^{*}$ Speaker. 


\section{Introduction}

The first-order nature of the deconfinement phase transition in SU(3) pure gauge theory is directly connected to the global Z(3) center symmetry and its spontaneous breaking [1, 2, 3, 4, 5]. The transition is eventually washed out by the explicit symmetry breaking induced by dynamical quarks. The strength of this breaking increases as the quark mass decreases. It is thus expected that the transition remains discontinuous in the heavy-quark region and becomes a continuous crossover at some critical value of quark mass [6]. This defines the critical end point (CEP) of the deconfinement phase transition.

Details of the phase structure of the deconfinement transition are revealed by examining the fluctuations of the Polyakov loop. Lattice results on these quantities exist for both the pure gauge theory [7] and the (2+1)-flavor QCD [8, 9]. In particular, the ratio of the transverse and longitudinal Polyakov loop susceptibility is motivated as an effective probe for deconfinement. In the case of pure gauge theory, it exhibits a $\theta$ function-like behavior at the critical temperature $T_{d}$, with almost no dependence on temperature on either side of the transition. In the presence of light quarks, the ratio is considerably smoothed, reflecting the crossover nature of the transition.

However, theoretical understanding of these quantities remains incomplete. It is therefore important to explore the properties of the Polyakov loop susceptibilities for different number of flavors, as functions of the quark mass in the heavy-quark region, thus bridging the gap between pure gauge theory and QCD.

In this work we discuss the phase structure of the deconfinement transition for heavy quarks. We study the behavior of the Polyakov loop susceptibilities near the CEP, and investigate their dependencies on the quark mass and quark chemical potential. We also relate the mean-field model results with lattice studies.

\section{Modeling deconfinement in the presence of quarks}

To explore the influence of heavy quarks on deconfinement, we consider the following effective model for the thermodynamic potential,

$$
T^{-4} \Omega=U_{G}\left(L, L^{*}\right)+U_{Q}\left(L, L^{*}\right)
$$

The $\mathrm{Z}(3)$ invariant part of the potential, $U_{G}$, is extracted from pure gauge theory. In this study the following phenomenological Polyakov loop potential is employed [9]:

$$
\begin{aligned}
U_{G}= & -\frac{1}{2} A(T) L^{*} L+B(T) \ln M_{H} \\
& +\frac{1}{2} C(T)\left(L^{3}+L^{* 3}\right)+D(T)\left(L^{*} L\right)^{2},
\end{aligned}
$$

where the $\mathrm{SU}(3)$ Haar measure $M_{H}$ is given by

$$
M_{H}=1-6 L^{*} L+4\left(L^{3}+L^{* 3}\right)-3\left(L^{*} L\right)^{2},
$$



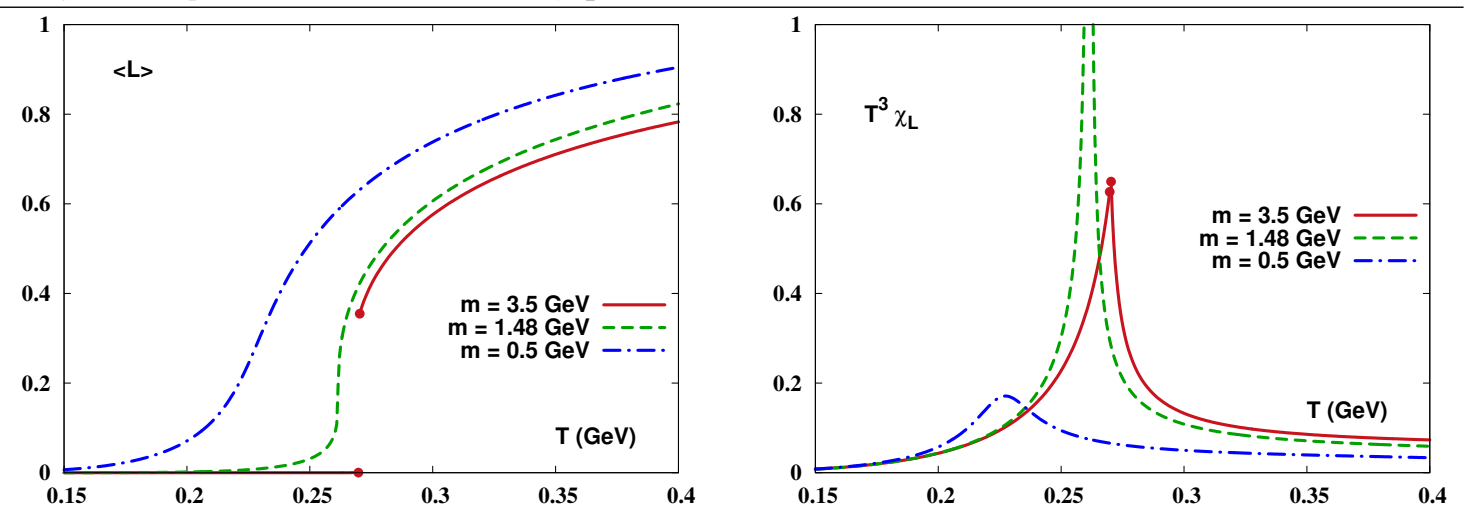

Figure 1: Temperature dependence of the thermal averaged Polyakov loop (left) and its longitudinal susceptibility (right) for different values of quark masses: $m=0.5,1.48$, and $3.5 \mathrm{GeV}$ at $N_{f}=3$.

and $A, B, C$, and $D$ are model parameters, tuned to describe lattice results on pure gauge thermodynamics. Particularly, this potential reproduces a first-order deconfinement phase transition at the critical temperature $T_{d}=0.27 \mathrm{GeV}$, which allows the expression of observables in physical units.

The explicit symmetry breaking term, $U_{Q}$, describes the coupling of quarks to the Polyakov loop. It can be obtained from the fermionic determinant in a uniform background gluon field as [10]

$$
\operatorname{det}\left(\hat{Q}_{F}\right)=\operatorname{det}\left(\left(-\partial_{\tau}+\mu-i g A_{4}\right) \gamma^{0}+i \vec{\gamma} \cdot \nabla-m\right)
$$

In the one-loop approximation, this gives the quark contribution to the effective Polyakov loop potential

$$
U_{Q}=-2 N_{f} \beta^{4} \int \frac{d^{3} k}{(2 \pi)^{3}}\left[T \ln g^{+}+T \ln g^{-}\right]
$$

where

$$
T \ln g^{+}=T \ln \left(1+3 L e^{-\beta E^{+}}+3 L^{*} e^{-2 \beta E^{+}}+e^{-3 \beta E^{+}}\right)
$$

specifies the coupling of quarks to the Polyakov loop, with $E^{+}=\sqrt{k^{2}+m^{2}}-\mu$. The function $T \ln g^{-}$describes the antiquarks, and is obtained from Eq. (2.6) by replacing $\mu \rightarrow-\mu$ and $L \leftrightarrow L^{*}$.

The thermal average of the Polyakov loop, $\ell=\langle L\rangle$ and its conjugate $\bar{\ell}=\left\langle L^{*}\right\rangle$, are obtained within the mean-field approximation as solutions of the gap equations

$$
\partial \Omega /\left.\partial L\right|_{L=\ell, L^{*}=\bar{\ell}}=0, \quad \partial \Omega /\left.\partial L^{*}\right|_{L=\ell, L^{*}=\bar{\ell}}=0
$$

In the following, we focus on how the position of the CEP changes with the number of quark flavors at vanishing and at finite quark density. 


\subsection{Deconfinement critical end point at $\mu=0$}

The expectation value of the transverse Polyakov loop, $L_{\mathrm{T}}=\left(L-L^{*}\right) /(2 i)$, vanishes due to the symmetry of the effective potential. Consequently, only the longitudinal Polyakov loop, $L_{\mathrm{L}}=$ $\left(L+L^{*}\right) / 2$, serves as an order parameter for deconfinement. On the other hand, the fluctuations of the order parameter along the longitudinal and transverse directions are both non-vanishing. They are quantified by the following susceptibilities [11]:

$$
\chi_{\mathrm{L}, \mathrm{T}}=\frac{1}{2} V\left[\left\langle L L^{*}\right\rangle_{c} \pm \frac{1}{2}\left\langle\left(L L+L^{*} L^{*}\right)\right\rangle_{c}\right] .
$$

where $\langle\ldots\rangle_{c}$ denotes the connected part. Within the mean-field model, $\chi_{\mathrm{L}, \mathrm{T}}$ are the inverse curvatures of the thermodynamic potential (2.1) along the respective directions around the global minimum $[9,11]$.

In Fig. 1 we show the Polyakov loop as a function of temperature for different values of quark masses. For a sufficiently large quark mass, the first-order nature of the phase transition persists, while at smaller quark masses, the explicit symmetry breaking increases resulting in the transition to be a crossover. The end point of the first-order transition line defines the critical value of the quark mass, $m_{\mathrm{CEP}}$.

To identify the CEP, we use the longitudinal fluctuations of the Polyakov loop. In Fig. 1 we show the longitudinal susceptibility for three degenerate quark flavors. While both susceptibilities depend on the value of the quark mass, only the longitudinal one shows an enhancement near the CEP [12]. The transverse susceptibility decreases monotonically with decreasing quark mass. Thus, for a given $N_{f}$, the CEP can be located by identifying the global maximum of $\chi_{\mathrm{L}}$. For different $N_{f}$, our model yields the following results for the critical quark masses,

$$
m_{\mathrm{CEP}}=1.10,1.35,1.48 \mathrm{GeV}, \text { for } N_{f}=1,2,3 .
$$

The resulting trend, with $m_{\mathrm{CEP}}$ increasing with $N_{f}$, agrees with the recent study in Ref. [13]. The location of the deconfinement critical end point is closely related to the form of the Polyakov loop potential [13]. In the present calculation, $U_{G}$ reproduces the lattice data on the equation of state as well as on the susceptibilities of the Polyakov loop. This feature is crucial for locating the CEP, which is influenced by fluctuations of the order parameter.

\subsection{Deconfinement CEP at finite chemical potential}

At finite $\mu$, the thermal averaged Polyakov loop $\ell$ and its conjugate $\bar{\ell}$ are both real, but in general different $[11,14,15]$. This is because at nonzero $\mu$ the effective action is complex $[11,15]$.

The finite density results for the longitudinal susceptibility is shown in Fig. 2.1. As in the case of $\mu=0$, only the longitudinal susceptibility is enhanced near the CEP, whereas the transverse susceptibility is insensitive to criticality.

The dependence of the critical quark mass $m_{\text {CEP }}$ on the chemical potential $\mu$ is shown in Fig. 2.1. The points in the figure are extracted from the divergence of $\chi_{\mathrm{L}}$, while the line is determined using only the leading explicit symmetry breaking term in the effective potential [12]. The increase of the critical quark mass with $\mu$ indicates that the first-order region shrinks with increasing density. This finding is consistent with the lattice results presented in Refs. [16, 17, 18]. 

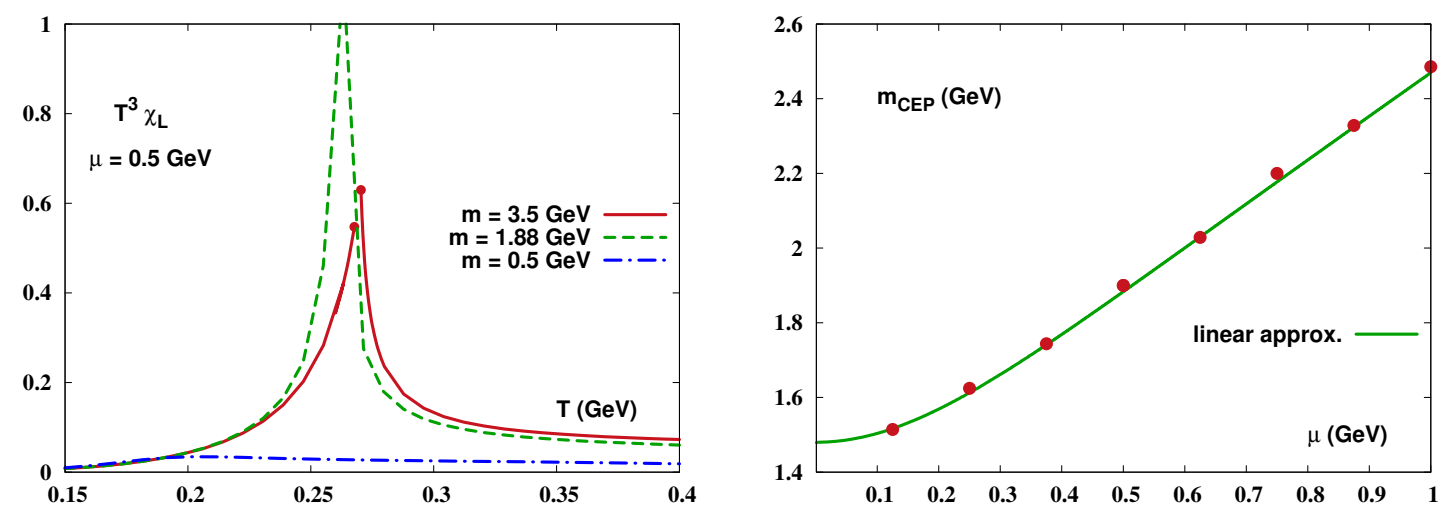

Figure 2: Left: The longitudinal $\chi_{\mathrm{L}}$ susceptibility (2.8) as a function of $T$ at $\mu=0.5 \mathrm{GeV}$ for different values of quark mass. Right: The critical quark mass as a function of the quark chemical potential for $N_{f}=3$. Solid points represent results obtained from the global maximum of $\chi_{\mathrm{L}}$. The line represents the result obtained by keeping only the linear symmetry breaking term in the quark potential [12].

\section{Interplay between confinement and chiral symmetry breaking}

In QCD, the expectation value of the Polyakov loop is substantially modified by dynamical quarks. A constructive way to explore deconfinement is to study various fluctuations associated with conserved charges. One of such key observables is the kurtosis of net-quark number fluctuations, which was proposed to be a measure of the onset of deconfinement [23].

In Fig. 3, the ratio $R_{A}=\chi_{A} / \chi_{R}$ is compared with the kurtosis of the quark number fluctuations, where $\chi_{A}$ represents the fluctuation of the modulus of the Polyakov loop [7].

We observe that quark deconfinement happens when $R_{A}$ undergoes a qualitative change. These abrupt changes in the Polyakov loop and quark number fluctuations appear in the same narrow temperature range around the pseudo-critical temperature of chiral symmetry restoration. This suggests that $T_{\text {deconf }} \simeq T_{\text {chiral }}$ at vanishing chemical potential. An extensive analysis for these observables is underway.

\section{Conclusions}

We have concentrated on the structure of the phase diagram and discussed the properties of the deconfinement critical end point within an effective model. The analysis was presented at vanishing and finite chemical potential for different number of quark flavors. It is shown that the considered model captures some of the basic properties of QCD in the heavy quark region, and yields results that can be compared with lattice calculations.

We have also argued, within lattice QCD studies, that the ratio of Polyakov loop susceptibilities characterizes the onset of deconfinement in QCD. We have compared the properties of this ratio with the kurtosis of the net baryon number fluctuations, which suggests that at vanishing quark chemical potential deconfinement appears in the region of the chiral crossover. 


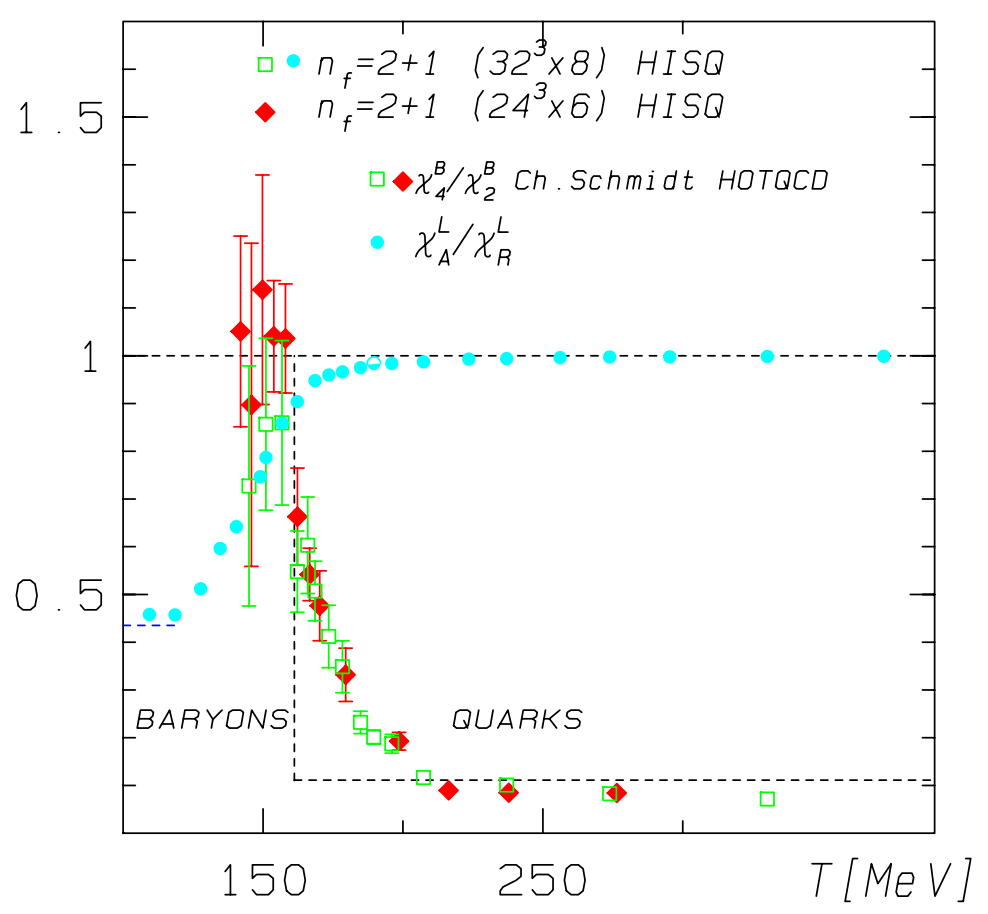

Figure 3: The ratio of the Polyakov loop susceptibilities $R_{A}=\chi_{A} / \chi_{R}$ and the kurtosis of net quark number fluctuations calculated on the lattice $[9,23]$

\section{Acknowledgments}

This work is partially supported by the Hessian LOEWE initiative through the Helmholtz International Center for FAIR (HIC for FAIR), and by the Polish Science Foundation (NCN), under Maestro grant 2013/10/A/ST2/00106.

\section{References}

[1] J. Greensite, "The Confinement problem in lattice gauge theory", Prog. Part. Nucl. Phys. 51, 1 (2003).

[2] G. 't Hooft, “On the Phase Transition Towards Permanent Quark Confinement”, Nucl. Phys. B 138, 1 (1978).

[3] L. G. Yaffe and B. Svetitsky, "First Order Phase Transition in the SU(3) Gauge Theory at Finite Temperature”, Phys. Rev. D 26, 963 (1982).

[4] L. D. McLerran and B. Svetitsky, "A Monte Carlo Study of SU(2) Yang-Mills Theory at Finite Temperature”, Phys. Lett. B 98, 195 (1981); "Quark Liberation at High Temperature: A Monte Carlo Study of SU(2) Gauge Theory”, Phys. Rev. D 24, 450 (1981).

[5] A. M. Polyakov, “Thermal Properties of Gauge Fields and Quark Liberation”, Phys. Lett. B 72, 477 (1978).

[6] F. Green and F. Karsch, "Mean Field Analysis of SU(N) Deconfining Transitions in the Presence of Dynamical Quarks", Nucl. Phys. B 238, 297 (1984). 
[7] P. M. Lo, B. Friman, O. Kaczmarek, K. Redlich, and C. Sasaki, "Probing Deconfinement with Polyakov Loop Susceptibilities”, Phys. Rev. D 88, 014506 (2013).

[8] C. McNeile, A. Bazavov, C. T. H. Davies, R. J. Dowdall, K. Hornbostel, G. P. Lepage and H. D. Trottier, "Direct determination of the strange and light quark condensates from full lattice QCD”, Phys. Rev. D 87, 034503 (2013).

[9] P. M. Lo, B. Friman, O. Kaczmarek, K. Redlich, and C. Sasaki, "Polyakov loop fluctuations in SU(3) lattice gauge theory and an effective gluon potential”, Phys. Rev. D 88, 074502 (2013).

[10] P. N. Meisinger, T. R. Miller, and M. C. Ogilvie, "Phenomenological equations of state for the quark gluon plasma”, Phys. Rev. D 65, 034009 (2002); P. N. Meisinger and M. C. Ogilvie, "Complete high temperature expansions for one loop finite temperature effects”, Phys. Rev. D 65 , 056013 (2002); P. N. Meisinger, M. C. Ogilvie and T. R. Miller, "Gluon quasiparticles and the polyakov loop", Phys. Lett. B 585, 149 (2004).

[11] C. Sasaki, B. Friman, and K. Redlich, "Susceptibilities and the Phase Structure of a Chiral Model with Polyakov Loops”, Phys. Rev. D 75, 074013 (2007).

[12] P. M. Lo, B. Friman, and K. Redlich, "Polyakov loop fluctuations and deconfinement in the limit of heavy quarks", Phys. Rev. D 90, 074035 (2014).

[13] K. Kashiwa, R. D. Pisarski and V. V. Skokov, "Critical end point for deconfinement in matrix and other effective models”, Phys. Rev. D 85, 114029 (2012).

[14] C. Ratti, M. A. Thaler, and W. Weise, "Phases of QCD: Lattice thermodynamics and a field theoretical model”, Phys. Rev. D 73, 014019 (2006).

[15] A. Dumitru, R. D. Pisarski, and D. Zschiesche, "Dense quarks, and the fermion sign problem, in a SU(N) matrix model”, Phys. Rev. D 72, 065008 (2005).

[16] S. Ejiri et al. [WHOT-QCD Collaboration], "Probability distribution functions in the finite density lattice QCD”, Proc. Sci. LATTICE 2012 (2012) 089.

[17] J. Langelage and O. Philipsen, “The deconfinement transition of finite density QCD with heavy quarks from strong coupling series”, J. High Energy Phys. 01 (2010) 089.

[18] M. Fromm, J. Langelage, S. Lottini, and O. Philipsen, "The QCD deconfinement transition for heavy quarks and all baryon chemical potentials", J. High Energy Phys. 01 (2012) 042.

[19] F. Karsch, E. Laermann, and A. Peikert, "Quark mass and flavor dependence of the QCD phase transition”, Nucl. Phys. B 605, 579 (2001).

[20] H. Saito, S. Ejiri, S. Aoki, T. Hatsuda, K. Kanaya, Y. Maezawa, H. Ohno, and T. Umeda, "Phase structure of finite temperature QCD in the heavy quark region”, Phys. Rev. D 84, 054502 (2011).

[21] C. Alexandrou, A. Borici, A. Feo, P. de Forcrand, A. Galli, F. Jergerlehner and T. Takaishi, "The Deconfinement phase transition in one flavor QCD”, Phys. Rev. D 60, 034504 (1999).

[22] T. A. DeGrand and C. E. DeTar, "Phase Structure of QCD at High Temperature With Massive Quarks and Finite Quark Density: A Z(3) Paradigm”, Nucl. Phys. B 225, 590 (1983).

[23] F. Karsch, S. Ejiri and K. Redlich, "Hadronic fluctuations in the QGP”, Nucl. Phys. A 774, 619 (2006). 